\title{
On Stellar Models with Blanketed Atmospheres as Boundary Conditions
}

\author{
Don A. VandenBerg ${ }^{1}$, Bengt Edvardsson ${ }^{2}$, Kjell Eriksson ${ }^{2}$, \\ Bengt Gustafsson ${ }^{2}$ and Jason W. Ferguson ${ }^{3}$ \\ ${ }^{1}$ Dept. of Physics and Astronomy, University of Victoria, Victoria, B.C., V8W 3P6, Canada \\ email: vandenbe@uvic.ca \\ ${ }^{2}$ Uppsala Astronomical Observatory, Box 515, SE-751 20 Uppsala, Sweden \\ email: be@astro.uu.se, Kjell.Eriksson@astro.uu.se, bg@astro.uu.se \\ ${ }^{3}$ Department of Physics, Wichita State University, Wichita, Kansas 67260-0032, U.S.A. \\ email: jason.ferguson@wichita.edu
}

\begin{abstract}
The impact on the predicted $T_{\text {eff }}$ scale of using the latest MARCS model atmospheres, instead of a fixed atmospheric structure (e.g., the gray $T-\tau$ relation) is examined. The former were fitted to stellar interior models at both the photosphere and at $\tau=100$ to determine the sensitivity of evolutionary tracks and isochrones for $[\mathrm{Fe} / \mathrm{H}]=0.0$ and -2.0 to the chosen fitting point. In the case of solar abundances, the $T_{\text {eff }}$ of the giant branch varied by up to 100-150 $\mathrm{K}$, depending on how the outer layers were treated. Much smaller variations were found for metal-poor giants (or main-sequence stars). Interestingly, models for the low solar $Z$ favored by Asplund et al. $(Z=0.0125)$ were unable to reproduce the gap near the turnoff in the C-M diagram of the old open cluster M 67, in contrast to models that assume $Z=0.0188$.
\end{abstract}

Keywords. Sun: abundances, stars: abundances, stars: atmospheres, stars: evolution

\section{Introduction}

It goes without saying that the interpretation of observations of stellar populations depends critically on the accuracy of the $T_{\text {eff }}$ scale of the stellar models that are used. Unfortunately, predicted temperatures are still quite uncertain due to their dependence on, among other things, low-temperature opacities, the treatment of convection, and the photospheric pressure (see, e.g., VandenBerg 1991). Of the various factors that impact computed temperatures, the role of the model atmosphere has been given the least attention. Most stellar models reported to date have determined the pressure at $T=T_{\text {eff }}$ by integrating the hydrostatic equation in conjunction with either the gray or a scaled-solar (e.g., Krisha Swamy 1966; hereafter KS66) T- $\tau$ relation (e.g., Yi et al. 2001, Pietrinferni et al. 2004, VandenBerg et al. 2006). There have been a few attempts to attach blanketed model atmospheres onto interior structures (e.g., Brocato et al. 1998, Cassisi et al. 2004), but it is not clear that both the atmosphere and interior models in such studies have assumed exactly the same chemical abundances, exactly the same treatment of convection, etc. Consequently, it is worthwhile to undertake the examination presented below in which the entire star is treated as consistently as possible.

\section{Stellar Models with Different Treatments of the Atmosphere}

Figure 1 compares various quantities as predicted by the Uppsala model atmosphere code (MARCS), for the region in the Sun between the photosphere (depth $=0.0$ ) and that radius where $\tau=100$, with those given by the Victoria stellar structure code. These calculations assume the Grevesse \& Sauval (1998) mix of heavy elements except 


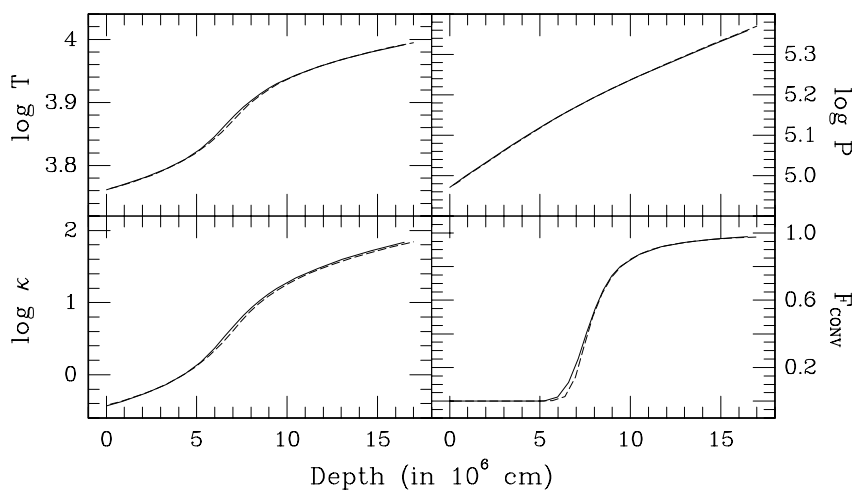

Figure 1. Comparison of various quantities in the sub-photospheric layers of the Sun as predicted by the MARCS atmosphere and Victoria interior codes (solid and dashed curves, respectively); see the text.
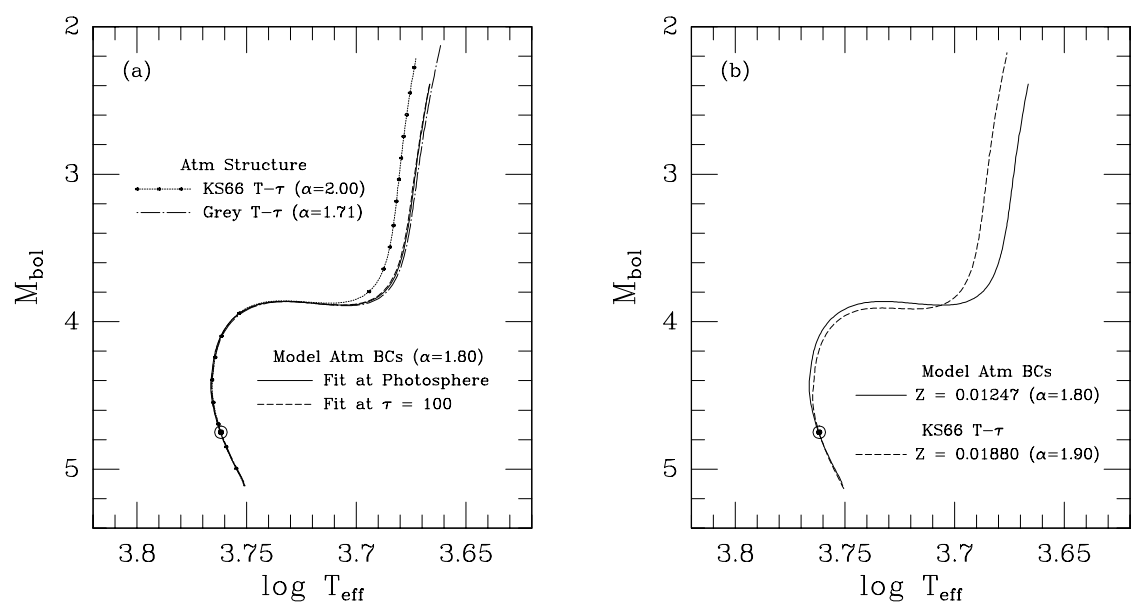

Figure 2. Panel (a): evolutionary tracks for a solar model for 4 different treatments of the atmosphere. The values of the mixing-length parameter needed to satisfy the solar constraint are as indicated (note the location of of the solar symbol). Panel (b): comparison of solar tracks for different assumed $Z$ values: that for $Z=0.0188$ is from VandenBerg et al. (2006).

as modified by M. Asplund and colleagues (e.g., see Asplund et al. 2005; and references therein), resulting in $Z=0.01247$, as well as the helium abundance $(\log N=10.949)$ and the value of the mixing-length parameter $(\alpha=1.80)$ that are needed to satisfy the solar constraint. In the case of the Victoria results, 3 of the usual 4 stellar structure equations (the luminosity is constant in the surface layers) were integrated inwards from the surface of the solar model assuming that the initial values of the dependent variables were the predicted photospheric pressure from the MARCS model along with the observed $T_{\text {eff }}$ and radius of the Sun. Although not shown, other quantities (e.g., $C_{P}, \rho, \nabla_{\text {ad }}, \mathrm{v}_{\text {conv }}$ ) are also in excellent agreement, which demonstrates that the physics implemented in the Uppsala and Victoria codes is nearly identical. (Some differences are expected because, e.g., the stellar interior code - but not the MARCS code — uses the diffusion approximation to the transfer equation.)

Figure 2a illustrates several evolutionary tracks applicable to the Sun when the atmospheric layers are described by either the KS66 or the gray $T-\tau$ relations and the 

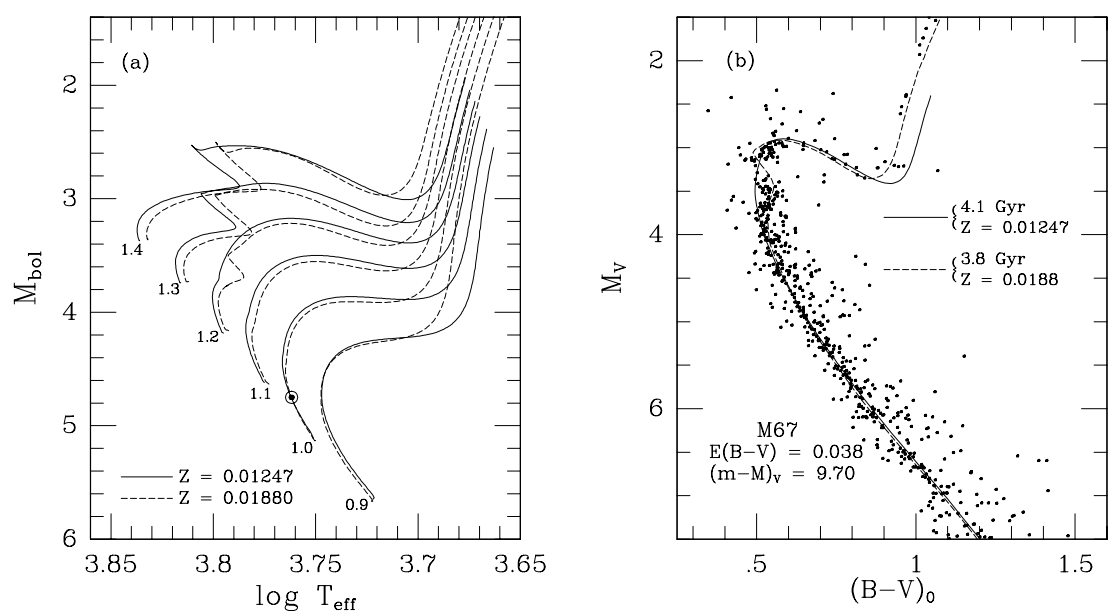

Figure 3. Panel (a): Evolutionary tracks for $Z=0.01247$ (this study) and $Z=0.0188$ (from VandenBerg et al. 2006) and the indicated masses, in solar units. Panel (b): Comparison of isochrones for the two $Z$ values and the indicated ages with the C-M diagram of M 67 (Montgomery et al. 1993). The assumed reddening and distance modulus are as noted.

photospheric pressure is determined by integrating the hydrostatic equation (i.e., $d P / d \tau=g / \kappa)$, or when MARCS model atmospheres are fitted to the stellar evolutionary models at either the photosphere or at $\tau=100$. Interestingly, except for the track using KS66 atmospheres, which is hotter than the rest (likely because of the large value of $\alpha$ that is needed to match the solar properties at the solar age), there is little to distinguish the four tracks that have been plotted. Worth emphasizing is the fact that the results appear to be essentially independent of where the model atmospheres are attached to the interior models (at least for this particular case). Calibrated solar tracks for two different metal abundances are illustrated in Figure $2 b$.

In fact, the assumed $Z$ of the Sun has important implications for the onset of core convection. In the case of the "Asplund" metallicity, the transition mass between stars that possess radiative cores throughout the main-sequence phase and those with convective cores is $\approx 1.195 \mathcal{M}_{\odot}$, whereas it is close to $1.145 \mathcal{M}_{\odot}$ if $Z=0.0188$ (see the tracks in Figure 3a). The open cluster M67, which is known to have very close to solar abundances (e.g., Tautvaisiene et al. 2000) provides an interesting test of the value of $Z$ in stars having $[\mathrm{Fe} / \mathrm{H}]=0.0$. As shown in Figure $3 \mathrm{~b}$, isochrones for the low $Z$ obtained by Asplund et al. for the Sun do not predict a turnoff gap where one is observed in M 67, while those for $Z=0.0188$ provide a very good match to the observed C-M diagram. (The treatment of the atmosphere has nothing to do with this difference: it is strictly a consequence of the amount of metals in the stellar interiors.) Although the present models do not allow for diffusive processes, a high $Z$ is apparently needed even if they are taken into account (see Michaud et al. 2004). It is not obvious whether the M 67 comparison is suggesting that the low solar $Z$ determined by Asplund et al. is incorrect, that there is a significant difference between the atmosphere and interior metallicities of the Sun, that our understanding of M 67 (or its turnoff stars) is somehow lacking, or ...

Figure $4 \mathrm{a}$ indicates that, at $[\mathrm{Fe} / \mathrm{H}]=-2.0$, the tracks for a $0.8 \mathcal{M}_{\odot}$ star are largely independent of the treatment of the stellar atmosphere. (The tracks using model atmospheres as boundary conditions do not extend all the way to the giant-branch tip because the MARCS atmospheres were not computed for sufficiently low gravities.) For these calculations, the "Asplund" $\log N$ abundances for most of the heavy elements were reduced 

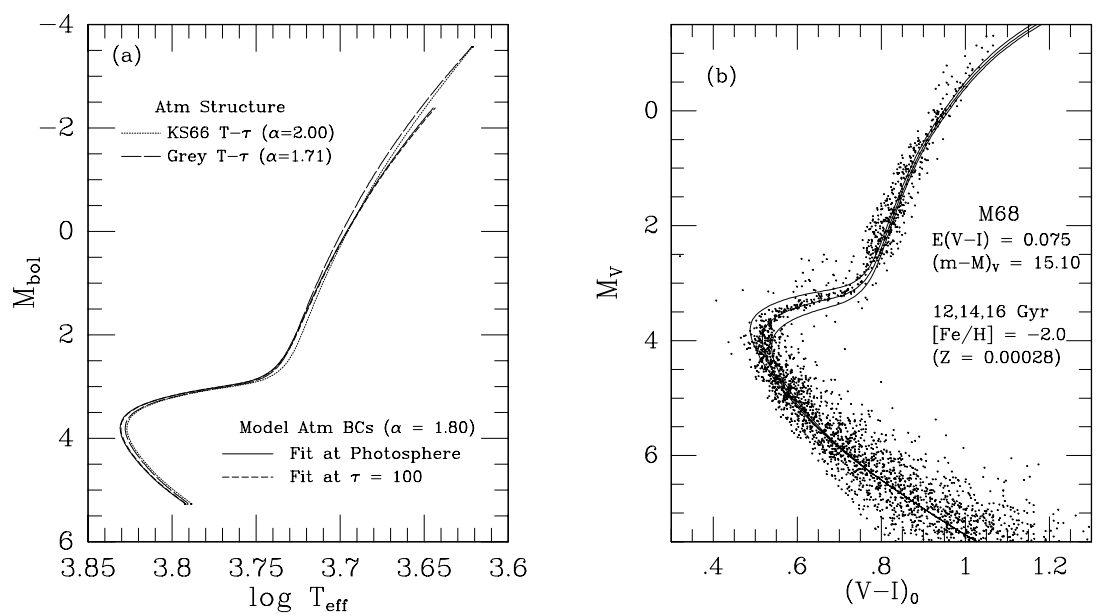

Figure 4. Panel (a): Similar to Fig. $2 \mathrm{a}$, except that the tracks are for a $0.8 \mathcal{M}_{\odot}$ star having $[\mathrm{Fe} / \mathrm{H}]=-2.0$. Panel (b): Fit of 12-16 Gyr isochrones to the $\mathrm{C}-\mathrm{M}$ diagram of the $[\mathrm{Fe} / \mathrm{H}]=-2.0$ (Carretta \& Gratton 1997) globular cluster M68, as obtained by Walker (1994).

by 2.0: a reduction of $\approx 1.6$ was assumed for the $\alpha$-elments so that $[\alpha / \mathrm{Fe}] \approx 0.4$ at low $Z$. It is evident in Figure $4 \mathrm{~b}$ that isochrones, specifically those employing MARCS model atmospheres, provide an excellent match to the observed C-M diagram of M68 on the assumption of very reasonable estimates of the distance and reddening.

\section{Conclusions}

The main results of this study are (1) attaching model atmospheres at the photosphere or at $\tau=100$ results in nearly the same $T_{\text {eff }}$ scale, at least for the cases considered here; (2) at both $[\mathrm{Fe} / \mathrm{H}]=0.0$ and -2.0 , the results obtained when the gray $T-\tau$ relation is used to describe the atmospheric layers are very similar to those which employ MARCS model atmospheres (due, perhaps, to compensating effects arising from differences in the assumed mixing-length parameters), and (3) the low $Z$ determined for the Sun by Asplund et al. appears to pose a problem for our understanding of M 67, or vice versa.

\section{References}

Asplund, M., Grevesse, N., Sauval, A.J., Allende Prieto, C., \& Blomme, R. 2005, A\&6A, 431, 693 Brocato, E., Cassisi, S., \& Castellani, V. 1998, MNRAS, 295, 711

Carretta, E., \& Gratton, R.G. 1997, A\&AS, 121, 95

Cassisi, S., Salaris, M., Castelli, F., \& Pietrinferni, A. 2004, ApJ, 616, 498

Grevesse, N., \& Sauval, A.J. 1998, Space Sci. Rev., 85, 161

Krishna Swamy, K.S. 1966, ApJ, 145, 174

Michaud, G., Richard, O., Richer, J., \& VandenBerg, D.A. 2004, ApJ, 606, 452

Montgomery, K.A., Marschall, L.A., \& Janes, K.A. 1993, AJ, 106, 181

Pietrinferni, A., Cassisi, S., Salaris, M., \& Castelli, F. 2004, ApJ, 612, 168

Tautvaisiene, G., Edvardsson, B., Tuominen, I., \& Ilyin, I. 2000, A\&A, 360, 499

VandenBerg, D.A. 1991, in: K. Janes (ed.), The Formation and Evolution of Star Clusters ASP Conf. Ser., 13, 183

VandenBerg, D.A., Bergbusch, P.A., \& Dowler, P.D. 2006, ApJ, 162, 375

Walker, A.R. 1994, AJ, 108, 555

Yi, S.K., Demarque, P., Kim, Y.-C., Lee, Y.-W., Lejeune, T., \& Barnes, S. 2001, ApJS, 136, 417 


\section{Discussion}

MeYnet: The metallicity provided by Asplund gives the present metallicity of the Sun. The Sun could have begun its life with a higher initial metallicity. The surface metallicity then has decreased due to diffusion. Do you account for that in your model?

VAndenBerg: Diffusion was not treated in the present models. Doing so would have resulted in a huge increase in the number of model atmospheres needed for this work as they would have had to allow for the stratification of element abundances arising from this process (which varies as a function of mass and age). However Michaud et al. (2004) have shown that, at an age of 4 Gyr diffusion would change the surface abundances of elements like oxygen by only $\sim 10 \%$ (much smaller than the change derived by Asplund et al. compared with previous estimates of solar abundances of CNO). Michaud's models also need high $\mathrm{Z}$ in order to predict a turnoff gap similar to what is seen in the M 67 CMD.

Peterson: It is puzzling that grey $\mathrm{T}-\tau$ and true model atmospheres give the same results here, since they do not for giant atmospheres. It is also suggested to try colors from Castelli et al. models, which have convective overshoot turned off

VANDENBERG: I agree that this is quite puzzling, but keep in mind that models using a grey $\mathrm{T}-\tau$ structure or blanketed atmospheres as boundary conditions require a different value of the mixing-length parameter, which has a remarkable capacity to compensate for variations in other physics. However, we plan to investigate this more fully before publishing this study in a refereed journal. Regarding Castelli colors: it is certainly worthwhile to make the comparisons you suggest, but I have relied on empirical $V-K$ vs. $T_{\text {eff }}$ relations which should be preferred over the color transformations from model atmospheres.

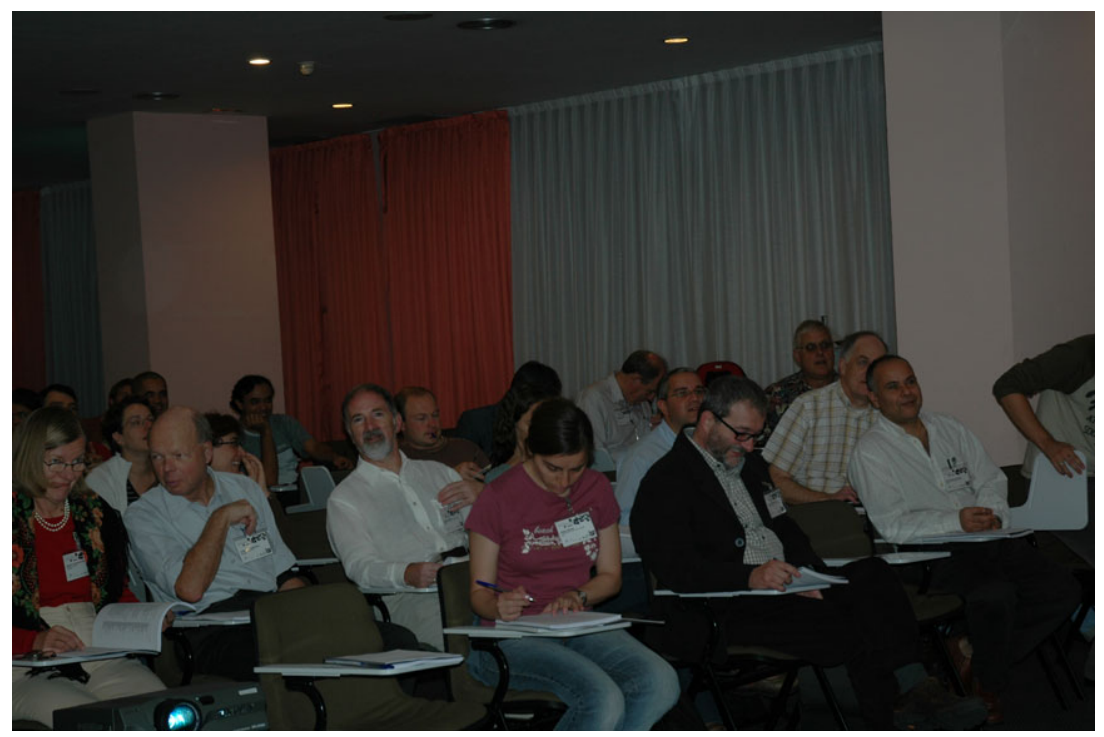

On the right of the picture (f.r.t.l.): Alexandre Vazdekis, the speaker, partly visible, Sandro Bressan, 